\title{
Comparison of Clinical Outcomes of Different Connection Modes of Extracorporeal Membrane Oxygenation Combine with Continuous Renal Replacement Therapy
}

\author{
Miaomiao Liu, MD,${ }^{1}$ Yang Yan, $\mathrm{MD},{ }^{1}$ Gang Li, MD, ${ }^{2}$ Ying Zhang, MD,${ }^{1}$ Fengwei Guo, $M D^{1}$ \\ ${ }^{1}$ Department of Cardiovascular Surgery, Xi'an Jiaotong University Medical College First Affiliated Hospital, China; ${ }^{2}$ Hanzhong 3201 \\ Hospital, China
}

\section{ABSTRACT}

Objective: To evaluate the effect of different connection modes of ECMO and CRRT on patients with acute kidney injury (AKI).

Methods: Twenty-one patients received ECMO with AKI. These patients were admitted to our center from December 2018 to February 2021, selected, and treated with both ECMO and CRRT. They were divided into A connection mode (pre-membrane-pre-pump connection) and B connection mode (post-membrane-post-pump connection). We compared clinical indicators and outcomes between two connection modes.

Results: There were eight cases $(38.91 \%)$ in A connection mode and 13 cases $(61.09 \%)$ in B connection mode, with median durations of ECMO assistance of 5 days and 7 days, respectively. Median flow rates of ECMO of $3.0 \mathrm{~L} / \mathrm{min}$ and $2.5 \mathrm{~L} / \mathrm{min}$, respectively; CRRT flow rates of $200 \mathrm{~mL} / \mathrm{min}$ and $180 \mathrm{~mL} / \mathrm{min}$, respectively. CRRT filter lifetime was over $48 \mathrm{~h}$ in both connection modes. Except for NT-pro BNP, no significant differences in clinical indicators were observed between the two groups before or after the treatment $(P>.05)$.

Conclusion: Both connection modes could achieve the therapeutic purpose and need no higher level of anticoagulation for patients simultaneously treated with ECMO and CRRT. Two modes had no impact on treatment effect and clinical indicators in patients. It had no effect on length of ICU stay and prognostic.

\section{INTRODUCTION}

In the last decade, extracorporeal membrane oxygenation (ECMO) technology has developed rapidly in the world and has been widely used in circulatory failure and/or respiratory failure [Hou 2020; Li 2019]. In adults undergoing ECMO,

Received October 7, 2021; accepted October 14, 2021.

Correspondence: Yang Yan, MD, Department of Cardiovascular Surgery, Xi'an Fiaotong University Medical College First Affiliated Hospital, 277 West Yanta Road, Xi'an, Shaanxi, P.R.China; +86(029)85323869 (e-mail: yanyang001001@163.com). the mortalities of acute kidney injury (AKI) are $78 \%$ and nonAKI patients only 20\% [Lin 2006]. Continuous renal replacement therapy (CRRT) is used in combination with ECMO for fluid management and simultaneity eliminated inflammatory substances and metabolites. To date, the method of CRRT and ECMO simultaneous application has been approved by most ECMO centers [Askenazi 2012]. However, the safest and most effective approach for connecting ECMO and CRRT has not been determined. When the CRRT circuit is connected to ECMO, its circuit pressure is nonphysiological under the high flow of ECMO. Thus, the inlet and outlet pressures of CRRT often deviate from the safe range, causing pressure alarms in CRRT and affecting the treatment effect [Askenazi 2012; Chen 2014]. Nevertheless, studies have reported different connection modes, using different standards and resulting in different conclusions [Seczyka 2014; Ricci 2010].

This study objective was to evaluate whether different connection modes of ECMO and CRRT have the impact on treatment effect or clinical indicators, such as blood routine examination, kidney functions and liver functions, and explore approaches to safely and effectively connect ECMO and CRRT equipment.

\section{MATERIALS AND METHODS}

\section{Connection methods}

There are many modalities to combine ECMO and CRRT, such as the separate vascular access of ECMO and CRRT, in-line connection of the hemodiafilter to the ECMO circuit, and connecting CRRT to ECMO in parallel circuit [Marlies 2018]. Among these, connecting ECMO and CRRT in parallel is the most common in clinical treatment. However, the use of different parallel modes of ECMO and CRRT may cause different problems. Further exploration of their impacts on treatment effect is necessary.

\section{Classification and discharge criteria}

A total of 21 patients received ECMO with AKI who all had been treated with ECMO and CRRT and who were admitted to our center from December 2018 to February 2021. The appropriate type of ECMO cannula was determined, according to each patient's condition. ECMO machine, tubes, and 
Table 1. General information on 21 patients with ECMO and CRRT

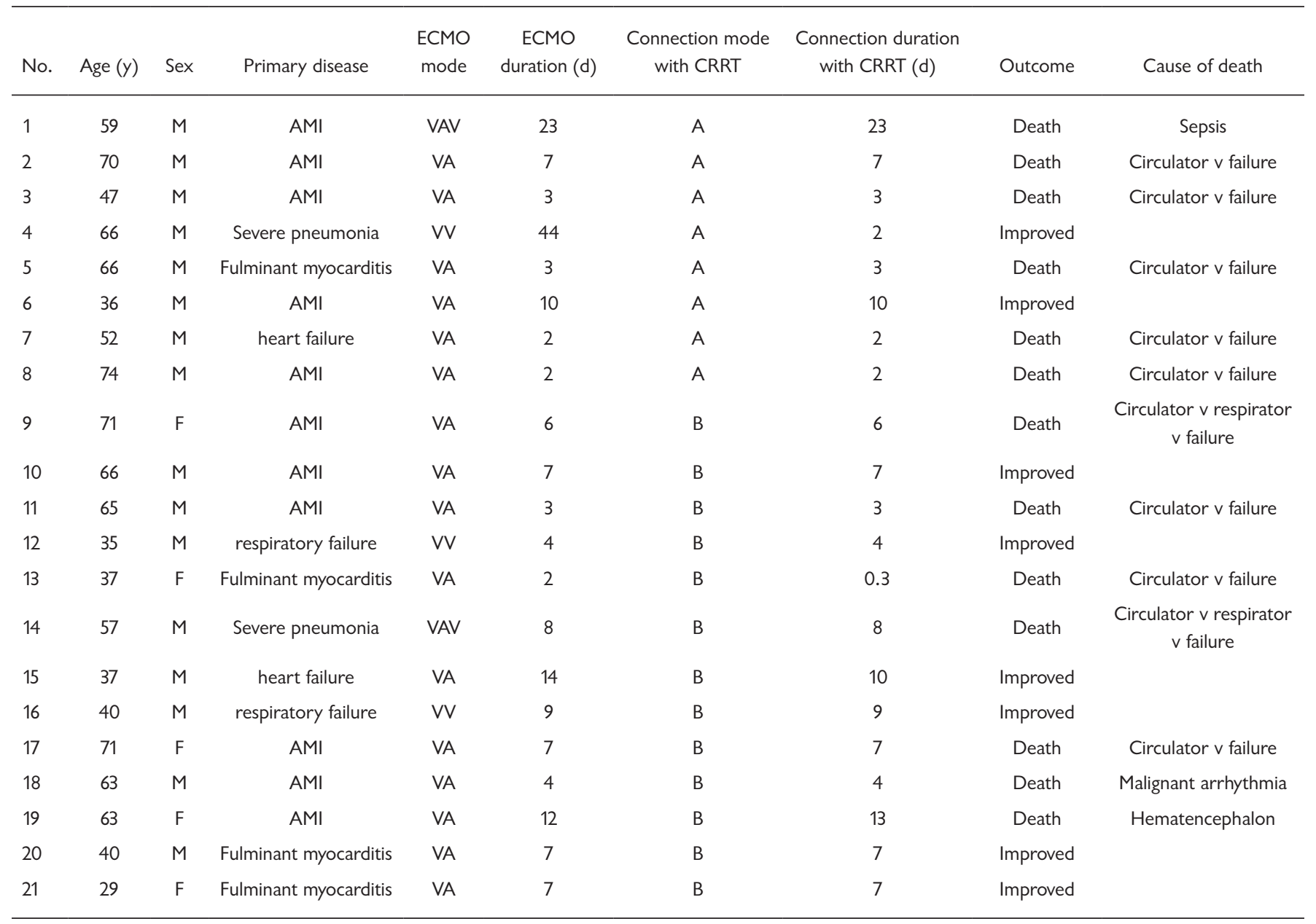

M, male; F, female; AMI, acute myocardial infarction; VA, veno-arterial mode; VV, veno-veno mode; VAV, veno-arterial-veno mode

membrane oxygenators were purchased from Maquet $\mathrm{GmbH}$ (Rastatt, Germany). CRRT machines were purchased from Baxter International (Deerfield, IL, USA) with ST-100 hemofilter and tubing. For each patient, we assembled the tube branches of these two machines into one of two parallel connection modes: pre-membrane oxygenator-pre-centrifugal pump or post-membrane oxygenator-post centrifugal pump. The 21 patients were divided into A connection mode (premembrane-pre-pump connection) and $\mathrm{B}$ connection mode (post-membrane-post-pump connection).

\section{The diagnostic criteria for AKI were in accordance with the Kidney Disease}

Improving Global Outcomes (KDIGO) 2012 Clinical Practice Guideline for the Evaluation and Management of Chronic Kidney Disease [Kellum 2013]. This means the patient's serum creatinine level had increased $\geq 26.5 \mu \mathrm{mol} / \mathrm{L}$ within 48 hours, and it had been confirmed or inferred that the creatinine value increased to $\geq 1.5$ times the baseline value within the past 7 days, or the patient's urine output was $<0.5 \mathrm{ml} / \mathrm{kg} / \mathrm{h}$ for 6 consecutive hours.

\section{Data collection}

This was a retrospective study. We collected patient demographic information, the ECMO and CRRT parameters, results from blood routine examination, kidney functions, liver functions, and biological indicators for infection. In addition, the ECMO assistance parameters, CRRT conditions parameters, total fluid balance, homeostasis times, infection conditions, length of ICU stay, and outcomes of the two groups of patients were compared to analyze the possible impact of the two modes on the patients.

\section{Statistical analysis}

In this study, variables distributed normally were presented as mean \pm standard deviation, and the difference between groups were compared with $\mathrm{T}$ test. Variables distributed nonnormally were presented as median (interquartile range, IQR), and Wilcoxon rank-sum test was used to analyze the differences between the groups. Categorical variables were summarized by frequency and proportion and tested by Fisher's exact test. $P<.05$ was considered statistically significant in this study. 
Table 2. Compare of the two connection modes on clinical data

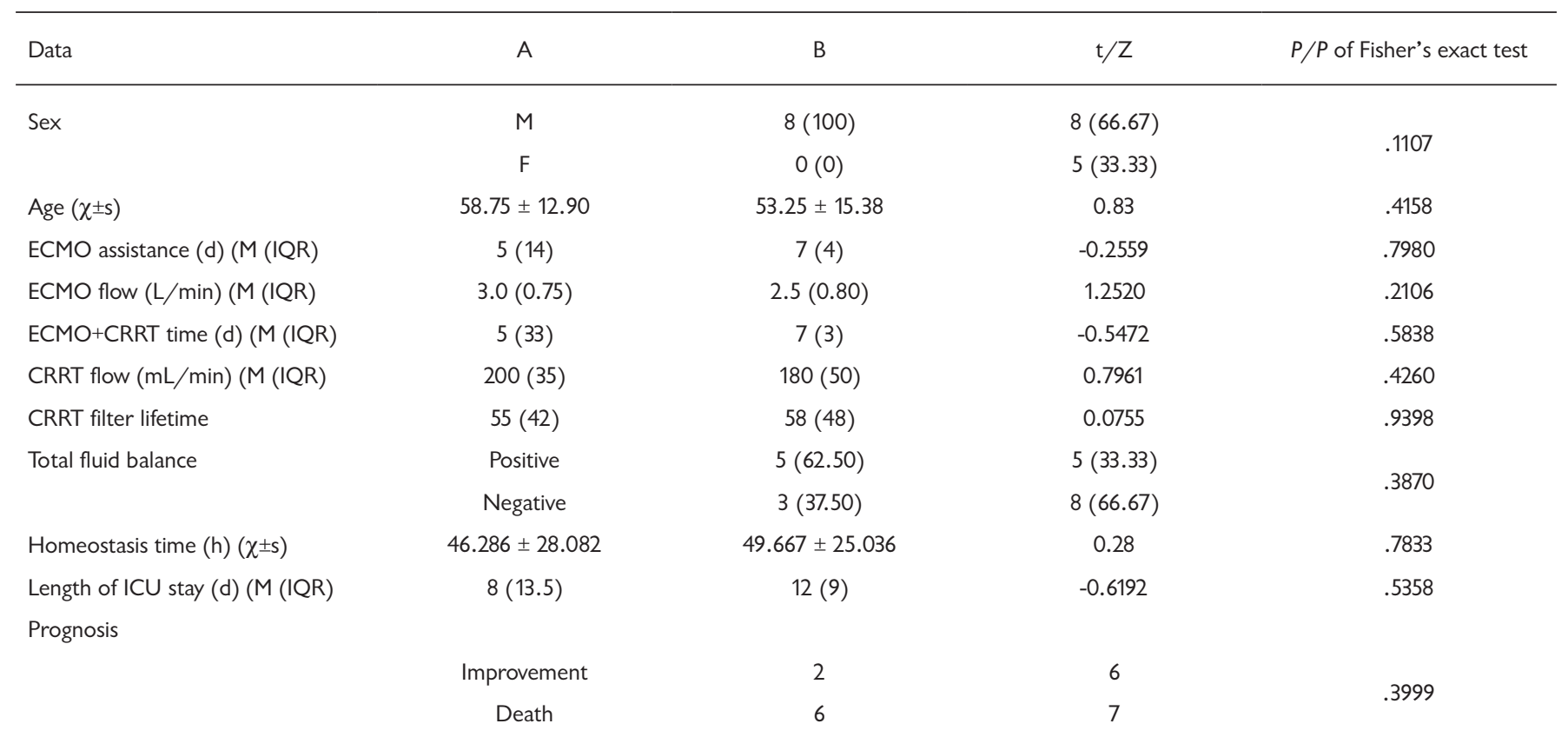

\section{RESULTS}

\section{General information}

Among the 21 patients treated with ECMO and CRRT in our center, there were 16 males $(76.19 \%)$ and five females (23.81\%), age $29-74$ years old, with primary diseases of acute myocardial infarction (11 cases, $52.38 \%$ ) and fulminant myocarditis (four cases, $19.05 \%$ ), including eight cases (38.91\%) with CRRT connected through pre-membrane-pre-pump connection in A connection mode and 13 cases (61.09\%) with CRRT connected through post-membrane-post-pump connection in $\mathrm{B}$ connection mode (Table 1).

\section{Comparison of the connection parameters}

The median duration of ECMO assistance in A connection mode and $\mathrm{B}$ connection mode was 5 days and 7 days, respectively. The CRRT flow rates of A connection mode and $\mathrm{B}$ connection mode were $200 \mathrm{~mL} / \mathrm{min}$ and $180 \mathrm{~mL} / \mathrm{min}$, respectively. The connection mode in A connection mode was prone to extremely negative CRRT pressure alarms, and the connection mode in $\mathrm{B}$ connection mode was prone to extremely positive CRRT pressure alarms. The median length of ICU stay for A connection mode and B connection mode was eight and 12 days, respectively. There were no significant differences found between the collected clinical data of the two groups, despite differences in the parameters of the two connection modes $(P>.05)$ (Table 2$)$.

Comparison of clinical test indices of different connection modes

By comparing the changes in related indicators before and after treatment between the two groups of patients, we observed a significant difference in the changes in $\mathrm{N}$-terminal NT-pro BNP levels before and after treatment. The change in A connection mode was $6553.57 \pm 10779.62 \mathrm{ng} / \mathrm{L}$, and the change in $\mathrm{B}$ connection mode was $-7168.96 \pm 14611.01$ $\mathrm{ng} / \mathrm{L}(\mathrm{t}=2.13, P=.0487)$. However, no significant differences in other indicators were found between the two connection modes before and after treatment $(P>.05)$ (Table 3).

\section{DISCUSSION}

ECMO-assisted patients often suffer from acute and critical illness, with an extremely high incidence of AKI that often requires concomitant CRRT. While ECMO assists circulatory and respiratory, CRRT performs fluid management, eliminates inflammatory mediators and metabolites, corrects internal environmental disorders [Fleming 2012], and possibly improves patient prognosis [Shum 2014]. The combination of ECMO and CRRT has been approved by various ECMO centers [Cao 2017].

Multiple connection methods are available for the combined use of ECMO and CRRT. Each can be used separately, but ECMO often requires systemic heparinization and prolonged clotting time to prevent thrombosis in the circuit. Under this circumstance, the invasive surgery for CRRT circuit placement may increase the risk of bleeding and infection and increase cost [Rubin 2010]. Further, the additional placement of CRRT circuit may also require deeper sedation or protective restraints on patients due to the reduction of CRRT alarms and the maintenance of longer filter usage, which may increase patient discomfort and complications [Seczyka 2014]. Therefore, most centers use CRRT in parallel with ECMO to achieve simultaneous application. This study showed that CRRT can safely and effectively be connected to the ECMO circuit in our two modes. Thirteen of the 20 patients achieved total fluid balance 
Table 3. Comparison of the two connection modes on clinical data $(x \pm s)$

\begin{tabular}{|c|c|c|c|c|}
\hline $\operatorname{Cr} \chi \pm s$ & $21.875 \pm 51.842$ & $3.462 \pm 90.781$ & 0.52 & .6086 \\
\hline GFR $\pm s$ & $-4.45 \pm 12.410$ & $4.527 \pm 34.242$ & -0.80 & .4376 \\
\hline NT-pro BNP $(\chi \pm s)$ & $6553.57 \pm 10779.62$ & $-7168.96 \pm 14611.01$ & 2.13 & .0487 \\
\hline $\operatorname{PLT}(\chi \pm s)$ & $-71.0 \pm 63.0465$ & $-79.7692 \pm 120.8795$ & 0.19 & .8523 \\
\hline WBC (M (IQR) & $4.21(11.54)$ & $-6.285(22.990)$ & 1.4466 & .1480 \\
\hline CRP (M (IQR) & $39.70(153.40)$ & $-17.20(113.80)$ & 0.000 & 1 \\
\hline PCT (M (IQR) & $0.37(14.117)$ & $-2.12(12.057)$ & 1.5045 & .1325 \\
\hline Infection & Positive & $2(25.00)$ & $4(30.77)$ & 1 \\
\hline AST (M (IQR) & $116.0(3731.0)$ & $-147.0(686.0)$ & 1.7743 & .0760 \\
\hline ALT (M (IQR) & $10.5(2152.0)$ & $-28.0(801.0)$ & 0.9777 & .3282 \\
\hline TBIL (M (IQR) & $31.40(60.30)$ & $3.50(45.5)$ & 1.8467 & .0648 \\
\hline Albumin $(\chi \pm s)$ & $2.3625 \pm 7.0795$ & $7.1923 \pm 9.6456$ & -1.22 & .2363 \\
\hline
\end{tabular}

Normal ranges: Cr, 41-73umol/L; BUN, 2.6-7.5mmol/L; GFR, >90mL/min; NT pro-BNP, 1-125ng/L; ALT, 9-50U/L; AST, 15-40 U/L; TBIL, 3.4-17.1umol/L;

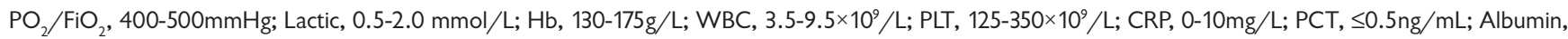
$40-55 \mathrm{~g} / \mathrm{L}$

or even negative balance with the aid of CRRT, resulting in the elimination of inflammatory metabolites and correction of the internal environment disorders. However, seven patients were critically ill with underlying diseases causing severely unstable circulation and respiratory conditions, which affected their treatment effect.

The various ECMO-CRRT connection methods have their advantages and disadvantages currently in use. In this study, we know that the A connection mode was prone to extreme negative pressure CRRT alarms, while the B connection mode was prone to extremely positive pressure CRRT alarms may cause of the direction of blood flow. When CRRT connect in ECMO circuit, the usage flow rate for ECMO is $2-5 \mathrm{~L} / \mathrm{min}$, which is 10 times than the usual blood flow rate for CRRT (commonly $0.15-0.2 \mathrm{~L} / \mathrm{min}$ ). Causing pressure alarms CRRT circuit to bear a larger pressure to cause the machine to stop and frequent stops reduced the treatment effectiveness and required circuit replacement due to blood clotting. The reduction of CRRT alarms and prevention of blood clotting in the filter is a particular challenge. By contrast, there was no significant differences in ECMO duration or flow, CRRT duration or flow and CRRT filter lifetime were found between the two connections. Both $\mathrm{A}$ and B connection could get the suggestion of KDIGO to keep the filtration fraction nearly $25 \%$ and the flow $35 \mathrm{ml} / \mathrm{kg} / \mathrm{h}$ [Jörres 2013]. CRRT filter lifetime was over $48 \mathrm{~h}$ in the two connection modes that systemic anticoagulation by heparin with ECMO met requirement of CRRT without adding more anticoagulants. The combination of ECMO and CRRT do not need a higher level of anticoagulation.

There have been many debates on the application timing and effects of ECMO combined with CRRT. Research has shown that CRRT effectively eliminates inflammatory mediators and metabolites and maintains the homeostasis of the internal environment with ECMO, providing circulatory and respiratory support [Cao 2017]. All 21 patients in this study reached homeostasis and improvement in liver functions, kidney functions, routine bloodwork, and infectious indicators, but there was no statistical difference. Thus, the connection modes had no alteration in treatment effect. Most reached a state of total fluid balance or even a negative balance, but their length of ICU stay was largely the same, showing no significant difference and prognostic alteration between the connection modes. It is consistent with the research by Antonucci [Antonucci 2013]. In addition, the two modes showed statistically significant differences in NT-pro BNP. It may be that the post-membrane-post-pump connection mode allows blood to pass through the membrane oxygenator of ECMO twice, which increases the elimination. Whether this finding is clinically meaningful requires further study.

This study showed that the connection modes of CRRT and ECMO in patients had no statistical difference in 
clinical data, but both modes could achieve the therapeutic purpose and did not need a higher level of anticoagulation. The connection modes had no effect on length of ICU stay and prognostic.

However, a limitation of this study is that the sample size may cause degrees of bias for further exploration of relevant research directions.

\section{REFERENCES}

Antonucci E, Lamanna I, Fagnoul D, Vincent JL, Backer DD, and Taccone FS. 2013. The impact of renal failure and renal replacement therapy on outcome during extracorporeal membrane oxygenation therapy. $\mathrm{J}$. Artif Organs 40, 746-754.

Askenazi DJ, Selewski DT, Paden ML, Cooper DS, Bridges BC, Zappitelli M, and Fleming GM. 2012. Renal replacement therapy in critically ill patients receiving extracorporeal membrane oxygenation. J. Clin J Am Soc Nephrol 7, 1328-1336.

Cao Y, Chai YF, Chen YG, and Emergency Clinical Application of Blood Purification Guideline Work Group. 2017. Expert consensus on clinical application of blood purification in China. J. Chinese Journal of Emergency Medicine 26, 24-36.

Chen H, Yu RG, Yin NN, and Zhou JX. 2014. Combination of extracorporeal membrane oxygenation and continuous renal replacement therapy in critically ill patients: a systematic review. J. Crit Care 18, 675.

Fleming GM, Askenazi DJ, Bridges BC, Cooper DS, Paden ML, Selewski DT, and Zappitelli M. 2012. A multicenter international survey of renal supportive therapy during ECMO: The Kidney Intervention During Extracorporeal Membrane Oxygenation (KIDMO) Group. J. ASAIO J $58,407-414$.

Hou XT. 2020. No ruly practice, no satisfied outcomes: final countdown for launching standardization of extracorporeal membrane oxygenation in China. J. Natl Med J China 25, 481-483.
Li CL, Hou XT, Hei FL, Qiu HB, Li A, Tong ZH, Li YM, Sun RQ, Liu B, and Jiang Y. 2019. China statistics of extracorporeal life support in 2018. J. National Medical Journal of China 99, 1911-1915.

Lin CY, Chen YC, Tsai FC, Tian YC, Jenq CC, Fang JT, and Yang CW. 2006. RIFLE classification is predictive of short-term prognosis in critically ill patients with acute renal failure supported by extracorporeal membrane oxygenation. J.Nephrol Dial Transplant 21,2867-2873.

Jörres A, John S, Lewington A, Wee PM, Vanholder R, Biesen WV, Tattersall J, and ad-hoc working group of ERBP. 2013. A European Renal Best Practice (ERBP) position statement on the Kidney Disease Improving Global Outcomes (KDIGO) Clinical Practice Guidelines on Acute Kidney Injury: part 2: renal replacement therapy. J. Nephrol Dial Transplant 28, 2940-2945.

Kellum JA, Lameire N, and KDIGO AKI Guideline Work Group. 2013. Diagnosis, evaluation, and management of acute kidney injury: a KDIGO summary (Part 1). J. Crit Care 17, 204.

Marlies O, Michael CJ, and Kianoush K. 2018. Continuous renal replacement therapy during extracorporeal membrane oxygenation: why, when and how? J. Curr Opin Crit Care 24, 493-503.

Ricci Z, Ronco C, and Picardo S. 2010. CRRT in series with extracorporeal membrane oxygenation in pediatric patients. J. Kidney Int 77, $469-470$

Rubin S, Poncet A, Wynckel A, and Baehrel B. 2010. How to perform a haemodialysis using the arterial and venous lines of an extracorporeal life support. J. Eur J Cardiothorac Surg 37, 967-968.

Seczyka B, Krikowski W, Nowak I, Jankowski M, Szułdrzyński K, and Szczeklik W. 2014. Continuous renal replacement therapy during extracorporeal membrane oxygenation in patients treated in medical intensive care unit: technica1 considerations. J. The Apher Dial 18, 523-534.

Shum H, Kwan AM, Chan KC, and Yan WW. 2014. The use of regional citrate anticoagulation continuous venous hemofiltration in extracorporeal membrane oxygenation. J. ASAIO J 60, 413-418. 